\title{
Factores que intervienen en el aprendizaje de la lectoescritura en los niños y niñas de Tasajera ${ }^{1}$ Factors that intervene in the learning of the
lecto-escritura in children and girls of Tasajera
}

DOI: http://dx.doi.org/10.17981/cultedusoc.9.2.2018.04

Fecha de recepción: 17/06/2018. Fecha de aceptación: 12/09/2018

Kelly Villegas-Effer ${ }^{2}$

Roberto Avendaño; Nubia Cáceres; Nelsy Charris; María López; Katheryn Palacios; Gilma Torres; Yomaira Ruiduiaz; Ana Bornachera; Dennys Caballero; Yulieth Maldonado; Elizabeth Cabana; Francisco Gutierrez; Nayibis Moreno; Alma Carbono y Venancio Serrano IED Rural De Tasajera, sede ERM La Gloria (Colombia) kvilla08@gmail.com

Para citar este artículo

Villegas-Effer, K., Avendaño, R., Cáceres, N., Charris, N., López, M., Palacios, K., Torres, G., Ruiduiaz, Y., Bornachera, A., Caballero, D., Maldonado, Y., Cabana, E., Gutierrez, F., Moreno, N., Carbono, A. y Serrano, V. (2018). Factores que intervienen en el aprendizaje de la lectoescritura en los niños y niñas de Tasajera. Cultura. Educación y Sociedad 9(2), 43-55. DOI: http://dx.doi.org/10.17981/cultedusoc.9.2.2018.04

\section{Resumen}

La presente investigación tuvo como objetivo Identificar los factores que intervienen en el aprendizaje de la lectoescritura en los niños y niñas de cuarto grado pertenecientes a la Institución Educativa Departamental Rural de Tasajera, jornada mañana sede número 2, La Gloria. El desarrollo del estudio se enmarcó en un diseño no experimental de tipo descriptivo, en el cual se observa el fenómeno tal y como se presenta en su ámbito natural para su posterior análisis, sin manipular intencionalmente sus variables, y se contextualiza en un estudio Transeccional donde los datos se recolectan en un único momento. Como técnica de recolección de información se utilizó la encuesta y como instrumento se aplicó un cuestionario y cuatro actividades pedagógicas. Como conclusiones se hace necesario desarrollar por el docente estrategias que promuevan el reforzamiento de los procesos que intervienen en el aprendizaje de la lecto-escritura, involucrando a toda la comunidad educativa.

Palabras Clave: Intervención, Aprendizaje, Lectoescritura.

\begin{abstract}
The objective of this research was to identify the factors that intervene in the learning of literacy in the fourth grade boys and girls belonging to the Rural Departmental Educational Institution of Tasajera, tomorrow morning, venue number 2, La Gloria. The development of the study was framed in a non-experimental design of descriptive type, in which the phenomenon is observed as it is presented in its natural environment for subsequent analysis, without intentionally manipulating its variables, and contextualized in a Transectional study where the data is collected in a single moment. As a technique for collecting information, the survey was used and a questionnaire and four pedagogical activities were applied as an instrument. As conclusions, it is necessary to develop by the teacher strategies that promote the reinforcement of the processes that intervene in the learning of the reading and writing, involving the whole educational community.
\end{abstract}

Keywords: Intervention, Learning, Literacy

\footnotetext{
${ }^{1}$ Este artículo ha sido derivado del Programa de Fortalecimiento de la Cultura Ciudadana y Democrática CT+I a través de la IEP apoyada en TIC en el Departamento de Magdalena: CICLÓN. Desarrollado con docentes miembros del Grupo de investigación "Investigando ando" pertenecientes a la Institución Educativa Departamental Rural de Tasajera, Sede ERM La Gloria.

${ }^{2}$ Líder del grupo de investigación "Investigando ando".
}

- The author; licensee Universidad de la Costa - CUC. 


\section{Introducción}

La lecto-escritura es un proceso por medio del cual propicia el aprendizaje. Permitirle utilizar al aprendiz las letras para representar sus ideas, sentimientos u otros, hace mas practico su aprendizaje. La lecto-escritura se debe concebir como una herramienta que les permitirá a los estudiantes aumentar su aprendizaje significativamente, lo cual es fundamental para su desarrollo integral, para tener una mejor calidad de vida en un mundo cada día más complejo y tecnificado. Es fundamental por parte del docente, especialmente en los primeros grados de educación básica fomentar en los educandos el proceso de lecto-escritura como un proyecto personal, eliminando la coacción, la inducción y el castigo. Esto implica que el niño se asuma como escritor, convirtiendo la lecto-escritura en un hecho personal e individual, para así poder obtener destrezas y habilidades.

El dominio de la lengua hablada y escrita permite realizar el proceso de apropiación de la cultura, conocimientos $\mathrm{y}$ técnicas de la sociedad en que vivimos en la actualidad. En la etapa de educación primaria las dificultades que afectan el proceso de la lecto-escritura, este dentro de las dificultades de aprendizaje en general, representa un alto porcentaje. El aprendizaje de la lecto-escritura, uno de los más alterados con frecuencia.

"Por tanto, es una adquisición básica, fundamental para los aprendizajes posteriores, de modo que los problemas específicos en ella, obstaculizan el progreso escolar de los niños que los experimentan. El estudiante con dificultades en lecto-escritura no sólo tiene problemas en el área de lenguaje, sino también en el resto de las áreas" (Bravo, 2001).
No tratar debidamente las dificultades de escritura y lectura en los tiempos adecuados, puede producir consecuencias negativas para los niños, ya sea a mediano o largo plazo, este puede influir en el fracaso social, educativo y/o profesional, incluso en la toma de decisiones en situaciones importantes de sus vidas.

Esta investigación se realiza con el fin de contribuir a mejorar la calidad educativa, puesto que la educación es un proceso integral y continuo donde los individuos se van desarrollando, es de gran importancia determinar desde las primeras etapas de formación del ser, cuáles podrían ser los aspectos que intervienen en el aprendizaje de la lecto-escritura.

La importancia de este proyecto se deriva de lo anteriormente expuesto, por cuanto permite a través de la investigación realizada presentar una propuesta en el próximo año escolar, que consiste en impulsar el desarrollo de una estrategia programática que contribuya a mejorar el rendimiento en escenarios escolares de los niños, específicamente en el área de lecto-escritura, de esta forma, el docente podrá contar con diversas estrategias metodológicas innovadoras en el proceso de la enseñanza de la lecto-escritura, que lo transforma en un ente activo y facilitador del aprendizaje, exponiendo la importancia del educando como el eje principal del proceso.

Algunas de las instituciones educativas carecen de identificación de las capacidades y habilidades de los estudiantes, para posteriormente ser aprovechadas, los docentes en sus metodologías de enseñanza deberían tener en cuenta la importancia del apoyo permanente necesitado por los estudiantes, esto podría dar mejores resultados en cuestiones de aprendizaje. Consideramos importancia de explotar al máximo todas las habi- 
lidades que tienen los estudiantes. Lo anterior produciría inmensos beneficios para la comunidad educativa. Este proyecto tiene como meta que cada niño evidencie la importancia de la lectura y la escritura. Que tenga una mira más clara al respecto, para que luego aprenda a quererla y sacarle provecho, que la perciba estratégicamente de una manera dinámica y satisfactoria para su vida; con esto se formaran niños con un alto nivel académico, puesto que la lectura y la escritura se constituye como una base fundamental del conocimiento.

La educación según (Rosseau, 2000), es "como un proceso continuo que empieza desde el nacimiento y sigue el desarrollo natural de las facultades latentes del ser humano: la sensación, memoria y la compresión"; por lo tanto, desde niño se debe llevar un proceso continuo que facilite el aprendizaje (Lorenzo, 2006). El sentido de esta investigación es identificar los procesos que intervienen en el aprendizaje de la lecto-escritura; y de este modo favorecer a la comunidad educativa, dada la necesidad existente teniendo en cuenta los bajos puntajes que presentan los estudiantes en pruebas externas y el bajo rendimiento académico de un porcentaje considerable de los mismos.

Desde el punto de vista práctico, es importante destacar que el éxito futuro del estudiante en su etapa educativa depende del adecuado desarrollo de sus habilidades en los primeros años de escolaridad, con relación a la eficaz utilización del lenguaje escrito y oral como herramienta principal en la comunicación con la sociedad. De esta manera, este estudio representa un aporte para la comunidad estudiantil en el sentido de que significa un referente teórico para los docentes de Educación Básica, para consultar y verificar los niveles de logro en función a la metodología para la enseñanza de la lecto-escritura, enriqueciendo la praxis conduciendo al niño o niña hacia el dominio de una lectura comprensiva y escritura clara y legible de todos los mensajes que desea transmitir, lo que indica la relevancia, utilidad individual, social y cultural del estudio.

Según Guerrero, (2016) "Los niños pasan por las mismas etapas de desarrollo, aunque éstas no se cumplen en la misma edad ni con el mismo ritmo. Las diferencias físicas, intelectuales y emocionales pueden ser más notorias. Es muy importante, por lo tanto, respetar profundamente la individualidad de cada niño." Todos somos diferentes física, intelectual y socialmente, pero debemos propiciar el libre desarrollo de la persona

\footnotetext{
"El niño debe adquirir una gran variedad de habilidades: capacidad de comunicarse, facultad de resolver problemas, de descubrir nuevas relaciones entre las personas y los objetos, entre objetos. Para ello requiere de un ambiente propicio y una relación cálida y amorosa." (Valverde, 2014).
}

Es nuestro deber con el niño: conocerlo, comprenderlo, apoyarlo y potenciarlo. Es una responsabilidad individual y colectiva: por este motivo se pretende aportar elementos Teóricos y prácticos que permitan evaluar, analizar, reconstruir y aportar. Analizando las actividades aplicadas y las encuestas logramos detectar fallas en el proceso de desarrollo de las competencias básicas de la lectura lectoescritura. Además, se puede decir que los objetivos propuestos en este proyecto son coherentes con el contexto específico de la Educación, por cuanto el proceso de aprendizaje de la lecto-escritura en los estudiantes, forma parte de los puntos fundamentales que se toman en consideración en el quehacer pedagógico del 
docente de primaria, el cual debe velar porque el niño o la niña adquieran habilidades instrumentales básicas para una lectura y escritura apropiada que le permita comunicarse sin dificultad.

La lecto-escritura es un proceso eminentemente individual, con el cual comprendemos lo simbolizado del texto y necesita del apoyo de la familia, la escuela y el ambiente sociocultural donde interactúa el estudiante. En este punto tienen gran importancia los canales de información, las bibliotecas, los clubes de lectura, las casas editoriales y el profesional de la docencia, quien debe proponer la búsqueda de métodos y estrategias para la enseñanza de la lengua, en concordancia se debe reflexionar constante y creativamente sobre el lenguaje y la importancia de éste para el desarrollo del ser humano y la sociedad.

(IEA), "afirma que la lectura tiene como finalidad entender cómo es el mundo y cómo ha sido y por qué las cosas funcionan de la manera en que lo hacen, y que los lectores pueden ir más allá de la adquisición de información y utilizarla para practicar el razonamiento y la acción" (citado en Pérez, 2007, p. 28). La lectura es un concepto de vital importancia dentro del sistema de la comprensión lectora. Echavarría (2006), comenta que "en la actualidad se conceptualiza a ésta como un proceso basado en el texto, de naturaleza interactiva, con propósitos específicos, y que depende tanto del texto como de la persona que lo lee."

Teniendo en cuenta que el presente proyecto de investigación se fundamenta en las categorías de trabajo colaborativo, estrategia didáctica y comprensión lectora, se hace necesario abordar una serie de aspectos relevantes para el desarrollo del fundamento teórico.
"La competencia lectora consiste en la comprensión y el empleo de textos escritos y en la reflexión personal. A partir de ello con el fin de alcanzar las metas propias, desarrollar el conocimiento y el potencial personal y participar en la sociedad activamente" (OCDE, 2013). Esto va más allá de interiorizar lo leído y/o escrito, sino enriquecernos intelectualmente adquiriendo todo el conocimiento posible.

\section{Trabajo Colaborativo}

El trabajo colaborativo, en un ámbito educativo, constituye un modelo de aprendizaje interactivo, que invita a los aprendices a construir juntos, para lo cual se debe conjugar esfuerzos, talentos y competencias mediante un plan de trabajo que les permitan alcanzar las metas establecidas concienzudamente. Como lo afirma Curiche, (2015) "más que una técnica, el trabajo colaborativo es considerado una filosofía de interacción y una forma personal de trabajo, que implica el manejo de aspectos tales como el respeto a las contribuciones individuales de los miembros del grupo."

En este sentido, este modelo de aprendizaje interactivo supone que las experiencias de aula deben superar el aprendizaje memorístico y mecanicista. Así como lo afirma Cabero, (2003) "Fomentar el trabajo colaborativo implica un cambio en la cultura escolar, en las prácticas de enseñanza/aprendizaje y en los procesos de evaluación; actualmente, la pedagogía y en sí, la educación, buscan que entre los estudiantes se fomente la colaboración entendida como una forma legítima y eficiente de producción tanto de conocimiento como de contenidos para que así, en la escuela, los estudiantes aprendan unos de otros". 
Esto implique los ambientes de aprendizaje enriquecidos por la aplicación del trabajo colaborativo permite que los estudiantes sean más activos en la medida que buscan la consecución de objetivos comunes desarrollando las habilidades y potencialidades de trabajo, asumen una democracia participativa asumiendo roles de grupo. El trabajo colaborativo como estrategia didáctica no implica "hacerle el trabajo al otro", ni confiar en los demás lo harán por mí, (Cabero, 2003), "El trabajo colaborativo, no resta responsabilidad a los actores, sino más bien hace esa responsabilidad más abordable al ser asumida como una misión en la que todos están comprometido a planificar, analizar y evaluar conjuntamente, cumpliendo cada quien lo que se requiere de su rol, fuera del trabajo conjunto"; con este entorno de aprendizaje se minimizan las tensiones entre los educandos y se motiva a una autorregulación del procesos mismo (Martínez, 2017; Martínez, 2014).

Desde esta perspectiva, se hace necesario que el docente asuma una actitud reflexiva y comprometida en la medida que genere estos ambientes colaborativos en el aula a fin que el estudiante participe activamente en la construcción del conocimiento. Dicha labor del docente la define Curiche, (2015), cuando señala que este debe realizar acciones concretas al estructurar planteamientos colaborativos: ser explícitos con los objetivos de la educación, asignar roles, adecuar o acondicionar el aula, en escenarios más comunicativos, crear una meta grupal de interdependencia positiva, evaluar la cantidad y la calidad de los alumnos.

Consecuente con lo planteado anteriormente, el docente debe conocer y apropiarse de la estrategia de trabajo colaborativo al momento de aplicarla en el aula de clases, atendiendo al desarrollo cognitivo y desarrollo social propio de la edad escolar de los estudiantes. Además, identificar las características de esta estrategia, aspectos que la integran y ventajas, "ya que el trabajo colaborativo es una estrategia en la que los participantes aprenden de manera significativa los contenidos, desarrollan habilidades cognitivas, además que contribuye a la formación de actitudes que van a contribuir en el desarrollo de cada persona" (Magallanes, 2011).

Con esta estrategia no solo se puede propiciar el incremento el rendimiento académico, sino también el desarrollo de otras virtudes como que el estudiante aprenda a trabajar en equipo, dar y recibir críticas, a planificar, a guiar y evaluar su actividad individual.

\section{Estrategias Didácticas}

Considerar el trabajo colaborativo como estrategia didáctica conlleva a reflexionar sobre la conceptualización de la estrategia didáctica en el aula. Por consiguiente, Carrasco (1997) haciendo alusión a estos dos conceptos, se refiere que "las estrategias en el campo didáctico son todos aquellos enfoques y modo de actuar que hacen que el profesor dirija con pericia el aprendizaje de sus alumnos. La estrategia didáctica pues, se refiere a todos los actos favorecedores del aprendizaje."

Sin embargo, teniendo en cuenta lo que expone carrasco, se debe tener en cuenta que todos los que permiten el desarrollo positivo de este proceso, no pueden ser exhibidos con exactitud, debido al carácter de "arte" de la didáctica, en otras palabras, la interpretación que el docente desarrolla de la práctica educativa es singular y contextualizada. Así 
mismo, los autores Velasco y Mosquera (2010), conceptualizan "la estrategia didáctica como la planificación del proceso de enseñanza aprendizaje para la cual el docente elige las técnicas y actividades que puede utilizar a fin de alcanzar los objetivos propuestos y las decisiones que debe tomar de manera consciente y reflexiva."

Del mismo modo, Carrasco (2004) señala que existen tres tipos de estrategias didácticas: Los métodos didácticos, las técnicas de enseñanza y los procedimientos didácticos; "en donde los métodos los define como la organización racional y práctica de los medios, técnicas y procedimientos de enseñanza, para dirigir el aprendizaje del estudiante es decir, ordenar y concretar un conjunto de reglas que permitan obtener los resultados esperados" (2004).

Las técnicas entonces, las describe como las acciones o estrategias, que se utilizan como herramientas aplicadas para hacer efectivo el método didáctico, y los procedimientos hacen parte de las técnicas de enseñanza y por lo tanto del método didáctico, en este sentido son los estímulos sobre los cuales el aprendiz desarrolla la gestión del conocimiento

Para Feo (2010), "el diseño de estrategias didácticas representa un eje integrador de los procedimientos que permiten al estudiante construir sus conocimientos a partir de la información que se suministra en el encuentro pedagógico; de esta afirmación se denota la importancia en la enseñanza y el aprendizaje escolar, además de la responsabilidad del profesor en el manejo de los elementos esenciales para su diseño.” Estos son los pilares fundamentales para un eficiente método de aprendizaje, para ser efectivo debe contar con una estrategia didáctica diseñada por el impartidor de conocimiento, además del uso correcto de las herramientas necesarias para su desarrollo y así logran los resultados esperados.

\section{Metodología}

La investigación se desarrolla dentro un marco cuantitativo, descriptivo que posibilita identificar características del objeto de estudio, establece formas de conducta y disposiciones del objeto de estudio, comprueba la relación entre categorías de investigación. De acuerdo con los propósitos planteados, el investigador señala el tipo de descripción que se propone realizar (Miguelez, 2006).

Según (Hernández, Fernández y Baptiste, 2006) "busca especificar las propiedades, características de las personas, grupos, comunidades, procesos, objetos o cualquier fenómeno que se someten a un análisis", es decir, mide y recolecta información de las variables.

\section{Diseño de Investigación}

En el contexto mencionado del punto anterior se decidió guiar la indagación a través de un diseño Transeccional y de campo, en el cual se hace necesario el trabajo desde la observación del fenómeno tal y como se presenta en su ámbito natural para su posterior análisis, sin manipular intencionalmente sus variables y recolectando los datos en un único momento, con el fin de describir y analizar sus incidencias e interrelaciones en dicho instante al momento del abordaje (Hernández, Fernández y Baptiste, 2006).

Igualmente, al estudiar la situación directa de la realidad, la investigación de campo se consideró de la misma manera como la define (Ander-Egg, 2006), quien la califica como toda indagación realizada sobre el terreno, para la cual se parte del 
proceso de recogida de datos primarios de una población, distribuida geográficamente, utilizando para tal propósito algunas técnicas que se aplican de forma directa a los individuos en estudio.

\section{Herramientas de recolección de Información}

Con el ánimo de dar respuesta a los objetivos planteados en la presente investigación, se desarrolló en el salón de clases con los estudiantes que participaron en el estudio, cuatro (04) actividades pedagógicas que se sustentaron en los indicadores y dimensiones establecidos en la revisión teórica, como son: conciencia fonológica, aumento del vocabulario, fluidez lectora y aumento del vocabulario. A su vez, buscando conocer el nivel de gusto por la lectura y nivel de compromiso con el proceso de aprendizaje de la lecto-escritura de las personas que participaron en la investigación, se utilizó la encuesta entendida como una técnica de recogida de información por medio de preguntas escritas y presentadas en un cuestionario; se seleccionó esta técnica porque permite abarcar el total de la muestra en menor tiempo y obtener respuestas específicas sobre la situación a investigar.

De acuerdo a la técnica se utilizó como instrumento un cuestionario dirigido a los estudiantes, docentes y padres de familia de $4^{\circ}$ de la jornada matinal, en su construcción se incluyeron 10 ítems para los padres de familia, 9 para los docentes y 8 para los estudiantes, cerrados y con alternativas de respuesta que oscilaban entre dos y cinco opciones posibles a saber. Cada respuesta representada con un número para efectos de facilitar el procesamiento de los datos. El instrumento se aplicó dentro de la institución, se solicitó permiso a los docentes y padres de familia para que permitieran el trabajo con los estudiantes. Se socializo el instrumento y se respondió en forma escrita.

\section{Población y muestra}

La población objeto de estudio fueron (15) padres de familia y (15) estudiantes de la Institución Educativa Departamental Rural Tasajera, para una muestra total de (30) participantes.

\section{Procedimiento}

Fase (1): Identificación de la problemática a estudiar.

Fase (2): Definición de la población y muestra.

Fase (3): Definición de las variables de estudio.

Fase (4): Fundamentación teórica.

Fase (5): Escogencia de las técnicas e instrumentos de recolección de información.

Fase (6): Aplicación de los instrumentos.

Fase (7): Análisis y procesamiento de los datos.

Fase (8): Generación de resultados.

Fase (9): Socialización y discusión de los hallazgos encontrados.

\section{Resultados}

En este artículo se presentan los resultados, el análisis y discusión derivados de las respuestas obtenidas con el instrumento aplicado a los estudiantes, padres de familia y docentes de $4^{\circ}$ de la Institución Educativa Departamental Rural Tasajera, del Municipio de Pueblo Viejo, Departamento del Magdalena; con el fin de identificar los procesos que intervienen en el aprendizaje de la lectoescritura. 


\section{Primer proceso evaluado:}

Conciencia fonológica

\section{Conciencia fonológica}

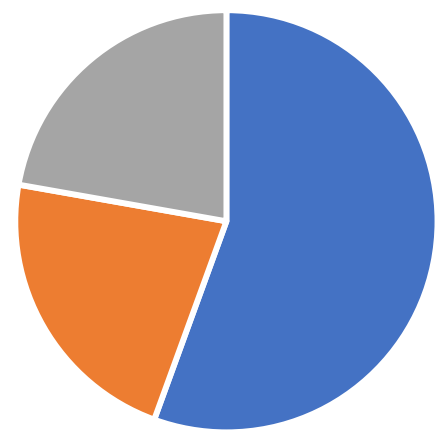

- 19 palabras 16 palabras 18 palabras

Actividad 1. El Muro de las silabas

En cuanto al indicador conciencia fonológica se puede evidenciar en la tabla que el $31.25 \%$ de los estudiantes logro conformar 19 palabras a partir de las sílabas, el $12.5 \% 16$ palabras y otro $12.5 \% 18$ palabras; lo cual quiere decir que más de la mitad de los estudiantes fue capaz de formar más de 15 palabras a partir de un grupo de sílabas. Según (Morais, 1987) los niños al desarrollar satisfactoriamente este proceso tienen la habilidad de descubrir y separar conscientemente los fonemas que componen las palabras. Lo que ellos denominarían como la conciencia segmental opera con el reconocimiento y el análisis de las unidades significativas del lenguaje, lo que facilita la transferencia de la información gráfica a una información verbal (Rivera, 2000).

Este proceso consiste en aprender a diferenciar los fonemas, en cuanto son expresiones acústicas mínimas e indispensables para que las palabras adquieran significado.
Segundo proceso evaluado: Aumento del vocabulario

Aumento del vocabulario

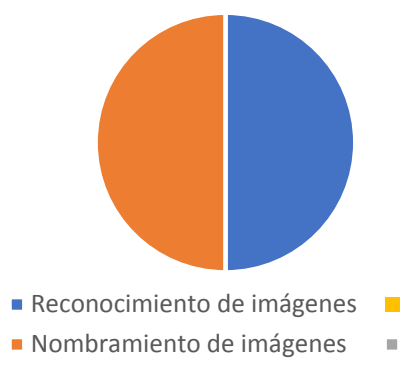

Actividad 2. Crucigrama.

En cuanto a la actividad 2 es notable que el $43.75 \%$ de los estudiantes reconoció 9 de las imágenes del crucigrama y logro llenarlo satisfactoriamente. Así mismo, otro $43.75 \%$ logro nombrar 10 de las imágenes del crucigrama. De acuerdo con Spender (1980), cuando vemos una palabra que conocemos o de las más utilizadas en nuestro vocabulario la leemos por la ruta visual que es la ruta "directa" y más rápida de la lectura. Es decir, que la mayoría de estudiantes que participaron de la investigación manejan ampliamente el vocabulario relacionado con las imágenes del crucigrama y esto les facilito su correcto diligenciamiento.

\section{Tercer proceso evaluado:} Fluidez Lectora

\section{Fluidez lectora}

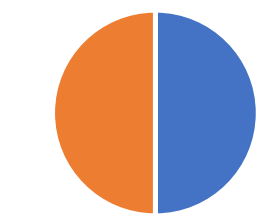

- Lectura fluida50,0

- Nivel de lectura -

Actividad 3. Lectura en pirámide. 
Considerando los resultados obtenidos a partir de la lectura que realizaron los estudiantes se logra evidenciar que el $50 \%$ no logra leer fluidamente, ósea que sobrepasaron el tiempo estimado de acuerdo a la lectura realizada. Así mismo, el otro $50 \%$ presento un buen nivel de lectura. Lo anterior, puede explicarse desde el concepto de Fillmore (citadp por Pradas, 2004) quien dice que «la fluidez es la habilidad de llenar el tiempo con habla y que una persona que es fluida en este sentido no tiene que pararse muchas veces a pensar qué es lo siguiente que quiere decir o cómo decirlo».

\section{Fluidez lectora}

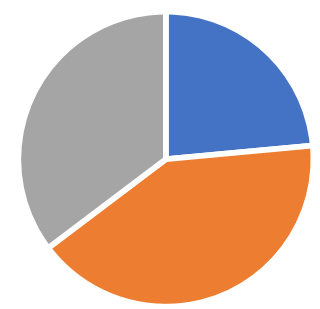

\footnotetext{
- Vocabulario - distincion de fonemas

- No reconocimiento de los signos de puntuacion

- lectura deoraciones completas
}

Siendo así el 50\% de los estudiantes que participaron de la investigación carece elementos como vocabulario, distinción de fonemas, lo cual dificulta la fluidez a la hora de leer. A esto es necesario agregarle que el $87.5 \%$ de los estudiantes no reconoce signos de puntuación al momento de realizar la lectura. Lo cual puede dificultar la comprensión del texto. Pero a su favor, es notable que un $75 \%$ no presenta lectura silábica, lo cual quiere decir, que un gran porcentaje de los estudiantes logra leer oraciones completas.
Cuarto proceso evaluado:

Conocimiento alfabético

Conocimiento alfabético

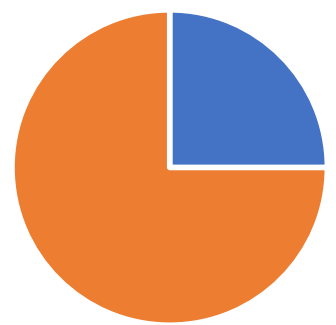

- Recoocimiento de letras

- Identificacion de palabras

Actividad 4. Sopa de letras.

De los procesos evaluados este es donde encontramos mayor dificultad, de acuerdo a los resultados obtenidos el mayor número de palabras que pudo encontrar un estudiante lo cual equivale al $6.25 \%$ a partir del reconocimiento de las letras del abecedario fue de 15 sobre 40. Y los porcentajes más altos que son $18.75 \%$ se ubican en 2 y 6 de palabras encontradas.

De acuerdo con (Orellana \& Bravo, 1999) hay estudios que muestran que el contacto de los niños con lo impreso (print) juega un rol importante en el aprendizaje del lenguaje escrito, debido a que genera un conocimiento visual de las formas ortográficas que facilita su reconocimiento posterior. El conocimiento de lo impreso permite a los niños, llegar a comprender el lenguaje escrito como un objeto visual que sirve para acceder a diferentes significados (Dourado \& Cardoso, 1998).

A través de una experiencia, muestran que el conocimiento del alfabeto capacita a los niños para aprender a leer procesando y guardando en la memoria las relaciones letra/ sonido en palabras. 
Tomando como referencia lo que dicen estos autores se podría anotar que es posible que los estudiantes que hacen pate de la investigación no estén muy relacionados con material impreso donde puedan reconocer las formas ortográficas y acceder a los diferentes significados en un determinado texto, "desarrollo de los procesos cognitivos de la lectura en alumnos normolectores y alumnos con dificultades especificas de aprendizaje" (Jiménez, 2010).

\section{Discusión}

Luego de realizar el análisis, discusión de los resultados y tomando en cuenta los objetivos de la investigación, se llegó a las siguientes conclusiones:

En relación al primer objetivo específico; Determinar los procesos que intervienen en el aprendizaje de la lectoescritura en los niños y niñas de grado 4 de la Institución Educativa Departamental Rural de Tasajera, jornada mañana sede número 2, La Gloria. Se utilizaron 4 actividades pedagógicas como instrumento para medir la variable a través de la dimensión procesos que intervienen en el proceso de aprendizaje de la lectoescritura, este instrumento se aplicó a los estudiantes de $4^{\circ}$ de básica primaria, encontrando que los indicadores conciencia fonológica y aumento del vocabulario, prevalecen en los estudiantes como parte del proceso de aprendizaje, lo cual, indica que los niños y niñas participantes en la investigación tienen la habilidad de "ver" que el lenguaje que oímos como un hilo sin fin está compuesto por palabras, sílabas, fonemas, rimas independientes entre sí. (Jiménez, 1992). Así mismo, en ellos se hace evidente que cuanto más vocabulario adquirimos mejor comprensión y fluidez lectora tenemos. Ya que cuando vemos una palabra que conocemos o de las más utilizadas en nuestro vocabulario la leemos por la ruta visual que es la ruta "directa” y más rápida de la lectura.

\begin{tabular}{ll}
\hline Población & \multicolumn{1}{c}{ Resultado } \\
\hline \multirow{2}{*}{$\begin{array}{l}\text { Encuesta } \\
\text { Estudiantes }\end{array}$} & $\begin{array}{l}\text { Teniendo en cuenta los resultados de la encuesta se puede observar que la gran mayoría } \\
\text { de los padres tienen un nivel académico satisfactorio. Lo cual hace que puedan ayudar a }\end{array}$ \\
& su hijo en el proceso de enseñanza aprendizaje. En su mayoría tienen 2 o 3 hijos así que, \\
& aunque tienen bastantes obligaciones gozan de algo de tiempo para realizar actividades \\
& que inciten a la lectura con sus hijos. Los padres manifiestan que les gusta leer, y a \\
diferencia de los niños la mayoría tiene clara cuál es la función de la biblioteca escolar.
\end{tabular}

Fuente: Elaboración propia. 
La fluidez se ve ralentizada tanto en niños como en adultos cuando nos encontramos con palabras poco utilizadas o desconocidas. Este conocimiento hace mención a la capacidad que tenemos los humanos para la conversión grafemafonema, y esto quiere decir que cuando vemos una palabra escrita sabemos distinguir que sonido corresponde a cada letra del alfabeto.

Por otra parte, en el segundo objetivo específico, Describir los procesos que intervienen en el aprendizaje de la lectoescritura en los niños y niñas de grado 4 de la Institución Educativa Departamental Rural de Tasajera, jornada mañana sede número 2, La Gloria se encontró que es necesario que los docentes fortalezcan los cuatro procesos en los que hace énfasis esta investigación, puesto que al momento de la aplicación de las actividades, se hizo notoria la necesidad que tenían los niños de una guía permanente para realizarlas, es decir que están muy acostumbrados a llevar a cabo los ejercicios y talleres de la mano del docente todo el tiempo, lo cual podría explicar porque cuando se enfrentan a pruebas externas cometen errores de forma como el diligenciar correctamente la hoja de respuestas. Es adecuado reforzar las actividades con material impreso, que le den a la menor independencia para trabajar bajo su propio ritmo de aprendizaje.

Con base en lo dicho anteriormente se hace necesario desarrollar por el docente estrategias que promuevan el reforzamiento de los procesos que intervienen en el aprendizaje de la lecto-escritura, involucrando a toda la comunidad educativa y tomando en consideración cuales son los procesos que prevalecieron en esta investigación. Por tal razón, el tercer objetivo de esta investigación cobra importancia, elaborar una propuesta tendiente a fortalecer el proceso de lecto-escritura utilizando las nuevas tecnologías de la información y la comunicación. Ya que más allá de evaluar unos resultados en un único momento, como docentes de la institución nuestro principal interés es mejorar la calidad educativa, y estamos convencidos que a partir de estos resultados podemos impulsar una propuesta basada en TICS para que los niños se enamoren de la lectura y la escritura, como también fortalezcan las debilidades que tengan a la fecha.

\section{Referencias}

Ander-Egg, E. (2006). Métodos y Técnicas de Investigación. Social_III. (Vol. III). Buenos Aires: Lumen Humanitas. Obtenido de https://www.google. com.co/search?q=ander+egg+2006\& oq $=$ ander+egg $+2006 \&$ aqs $=$ chrome. . 69i57.2270j0j7\&sourceid=chrome\&i $\mathrm{e}=\mathrm{UTF}-8$.

Avendaño, I., Cortés, O., Guerrero, H. (2015) Competencias sociales y tecnologías de la información y la comunicación como factores asociados al desempeño en estudiantes de básica primaria con experiencia de desplazamiento forzado. Diversitas: Perspectivas en Psicología, 11(1). 13-36 Obtenido de http://www.scielo.org.co/ pdf/dpp/v11n1/v11n1a02.pdf.

Bravo, L. (2001). Psicología de las dificultades del aprendizaje escolar (Séptima ed.). Santiago de Chile: Universitaria S.A. Obtenido de http:// biblioteca.salamandra.edu.co/libros/ Psicologia\%20de\%20las\%20dificultades\%20del\%20aprendizaje\%20escolar.pdf.

Carrasco, J. (2004). Una didáctica para hoy: cómo enseñar mejor. Ediciones Rialp. 
Cabero, J. (2003). Principios pedagógicos, psicológicos y sociológicos del trabajo colaborativo: su proyección en la telenseñanza. En, Redes de comunicación en la enseñanza: las nuevas perspectivas del trabajo corporativo (129-156). Paidós Ibérica.

Curiche, D. (2015). Desarrollo de habilidades de pensamiento crítico por medio de aprendizaje basado en problemas y aprendizaje colaborativo mediado por computador. [Tesis inédita de maestría]. Universidad de Chile, Santiago de Chile.

Dourado, \& Cardoso. (1998). La enseñanza del lenguaje escrito en un modelo interactivo. Obtenido de https://sites. google.com/site/adquisiciondelalectura/modelo-interac/6-aprendizaje-delcodigo-y-del-principio-alfabetico.

Echavarria, M. C. (1985). Desarrollo ya atención en niños de 7 a 12 años. San José de Costa Rica: Editorial Universidad Estatal a Distancia.

Feo, R. (2010). Orientaciones Básicas para el Diseño de Estrategias Didácticas. Tendencias Pedagogicas (16), 219-236.

Guerrero. H. y Cepeda, M. (2016). Uso de estrategias pedagógicas para el fortalecimiento de la convivencia escolar de jóvenes vulnerables. Obtenido de http://saber.ucv.ve/ojs/index.php/ rev_ped/article/view/12501.

Jiménez, J. E. (1992). Metaconocimiento fonológico: estudio descriptivo sobre una muestra de niños prelectores en edad preescolar. Infancia y aprendizaje, 57. 49-66.

Jiménez, J. E. (2010). Desarrollo de los procesos cognitivos de la lectura en alumnos normolectores y alumnos con dificultades especificas de aprendizaje. Obtenido de http://www.ince.mec.es/ revistaeducacion/re353/re353_13.pdf.
Lorenzo, J. R. (2006). Principio alfabético y aprendizaje de la lectura. Argentina. Obtenido de https:// ansenuza.unc.edu.ar/comunidades/bitstream/handle/11086.1/739/ Principio\%20alfab\%C3\%A9tico\%20 y\%20aprendizaje\%20de $\% 201 \mathrm{a} \% 20$ lectura.pdf?sequence $=1$.

Martinez, J. C., Berthel, Y., \& Vergara, M. S. (2017). Síndrome de Burnout en profesores y su relación con el aprendizaje de los estudiantes de básica primaria de una institución educativa oficial de Sincelejo (Colombia), 2016. Revista Salud Uninorte, 33 (2) .118-128. http://dx.doi. org/10.14482/sun.33.2.10538

Martínez, J. (2014). Modelo de evaluación del Proceso de Atención de Enfermería. [Tesis doctoral]. Universidad del Atlántico, Atlántico, Barranquilla. http://dx.doi. org/10.13140/RG.2.2.35792.00000

Miguelez, M. (2006). La investigaciópn cualitativa (síntesis conceptual). Revista II PSI, 09, 123-146. Obtenido de Http://sisbib.unmsm.edu. pe/bvrevistas/investigacion_psicologia/v09_n1/pdf/a09v9n1.pdf.

Morais, A. y. (1987). Por un enfoque psicolinguistico del aprendizaje de las lecturas y sus dificultades. Obtenido de http://www.um.es/langpsy/ Docencia/Lenguaje/Practicas/Dislexia/Alegria.1985.IA.pdf.

Orellana, E., \& Bravo, L. (1999). Procesos predictivos del aprendizaje inicial de la lectura en primer año básico. Obtenido de https://biblat. unam.mx/pt/revista/boletin-deinvestigacion-educacional/articulo/ procesos-predictivos-del-aprendizaje-inicial-de-la-lectura-en-primerano-basico. 
Pradas, M. (2004). La fluidez y sus pausas: enfoque desde la interpretación de conferencias. Granada: Comares. Obtenido de https://cvc.cervantes.es/ensenanza/ biblioteca_ele/publicaciones_centros/ PDF/rio_2008/37_horche-marco.pdf

Rivera, I. D. (2000). La enseñanza de la Lectoescritura. Obtenido de http://114. red-88-12-10.staticip.rima-tde.net/mochila/sec/monograficos_sec/ccbb_ceppriego/lengua/infantil/ensenanza_lectoescritura.pdf.

Hernández, R., Fernández, C. y Baptiste, P. (2006). Metodología de la investigación (4 ed.). México, D.F.: McGraw-Hill. Obtenido de https://investigar1.files. wordpress.com/2010/05/1033525612mtis_sampieri_unidad_1-1.pdf
Rosseau, J.-J. (2000). La educación en J.J. Rousseau: un antecedente metodológico de la enseñanza basada en la. Obtenido de https://revistas.ucm. es/index.php/RCED/article/viewFile/42646/44231

Valverde, Y. (2014). Lectura y escritura con sentido y significdo, como estrategia pedagógica en la formación de maestros. Obtenido de http://www. actiweb.es/educadora_andrea_reyes/archivo6.pdf 